\title{
Heart Failure Duration Combined with Left Atrial Dimension Predicts Super-Response and Long-Term Prognosis in Patients with Cardiac Resynchronization Therapy Implantation
}

\author{
Zhinian Guo, Xiaoyan Liu, Chuan Liu, Jie Yang, Xiaofeng Cheng, Yunlong Chen, \\ Ping Li, Yongming He, and Jiang Wang $\mathbb{D}$ \\ Department of Cardiology, Xinqiao Hospital, Army Medical University (Third Military Medical University), \\ 183 Xinqiao Street, Chongqing 400037, China
}

Correspondence should be addressed to Jiang Wang; 1530579054@qq.com

Received 21 March 2019; Accepted 15 May 2019; Published 24 June 2019

Academic Editor: Michael Gotzmann

Copyright (C) 2019 Zhinian Guo et al. This is an open access article distributed under the Creative Commons Attribution License, which permits unrestricted use, distribution, and reproduction in any medium, provided the original work is properly cited.

\begin{abstract}
Background. Response to cardiac resynchronization therapy (CRT) varies significantly among patients. This study aimed to identify baseline characteristics that could predict super-response to CRT and to evaluate the long-term prognosis in super-responders. Methods. We retrospectively reviewed the data of 73 consecutive patients who received CRT. Patients were considered as superresponders after 6-month follow-up when NYHA class reduction to I or II combined with left ventricular ejection fraction (LVEF) $\geq 50 \%$ was observed. Patients were divided into super-responders group and non-super-responders group. All-cause mortality or hospitalization for heart failure (HF) was referred to the combined end point. Results. 17 (23.3\%) patients were super-responders. HF duration, left atrial dimension (LAD), and left bundle branch block (LBBB) were independent predictors of super-response to CRT. The combination of HF duration and LAD could provide more robust prediction of super-response than standalone HF duration ( 0.899 versus $0.789, \mathrm{Z}=2.207, \mathrm{P}=0.027)$ or standalone $\mathrm{LAD}(0.899$ versus $0.775, \mathrm{Z}=2.487, \mathrm{P}=0.013)$. super-responders had excellent LV reverse remodeling. The cumulative incidences of combined end point were significantly lower in the superresponders group, $\mathrm{LAD} \leq 42 \mathrm{~mm}$ group, and combination of $\mathrm{HF}$ duration $\leq 48$ months and $\mathrm{LAD} \leq 42 \mathrm{~mm}$ group. LBBB remained associated with a lowered risk of the combined end point (HR: 0.19, 95\% CI: 0.07-0.57, P = 0.003), whereas LAD was associated with a raised risk of the combined end point (HR: 1.09, 95\% CI: 1.02-1.17, P = 0.014). Conclusions. HF duration, LAD, and LBBB independently predicted super-response. The combination of HF duration and LAD makes more robust prediction of CRT superresponse. Super-responders had excellent LV reverse remodeling and decreased the incidences of the combined end point. LBBB and LAD were independently associated with the combined end point.
\end{abstract}

\section{Introduction}

Cardiac resynchronization therapy (CRT) has become standard therapy for heart failure (HF) patients with left bundle branch block (LBBB) and prolonged QRS duration except optimum medical therapy [1]. It not only improves cardiac function, HF symptoms, and quality of life, but also reduces morbidity and mortality $[2,3]$. However, response to CRT varies significantly among patients. Unfortunately, 30\%-45\% of patients fail to demonstrate improvement in cardiac function, left ventricular function (LVEF), or left ventricular (LV) remodeling after CRT[2]. Nevertheless, certain patients realize near normalization or normalization of LVEF, referred to as "super-responders", was observed in approximately $12 \%-37.8 \%$ of CRT recipients [3-6].

Hence, it is of clinical importance to identify baseline characteristics which are predictive of super-response. It has been reported that shorter duration of HF symptoms, smaller $\mathrm{LV}$, smaller left atrial dimension (LAD), LBBB, wider QRS, and nonischemic cardiomyopathy (NICM) are associated with super-response $[5,7,8]$. However, the definitions of super-response vary in previous studies, with different predictors of super-response to CRT reported. 
The aim of the present retrospective study is (1) to identify baseline characteristics that could predict super-response to CRT, (2) to evaluate the value of independent predictors in predicting super-response, and (3) to analyze the long-term prognosis in super-responders.

\section{Materials and Methods}

2.1. Study Patients. This single center, retrospective study enrolled 73 consecutive patients who received CRT with or without an implantable defibrillator (CRT-P/D) from June 2014 to December 2017. The inclusion criteria were as follows: (1) New York Heart Association (NYHA) classes II to IV despite optimal medical therapy [angiotensin-convertingenzyme inhibitors (ACE-I) or angiotensin receptor blockers (ARB), $\beta$-blockers, and aldosterone antagonists] $\geq 3$ months, (2) left ventricular ejection fraction (LVEF) $\leq 35 \%$, and (3) QRS duration $\geq 130 \mathrm{~ms}$. Patients with narrow QRS, myocardial infarction $\leq 3$ months previously, or optimal medical therapy $\leq 3$ months were excluded from this study. The study was approved by the Clinical Research Ethics Board at the Third Military Medical University. All participants volunteered to participate in the study and signed informed consent.

2.2. Echocardiography. Echocardiography was performed at baseline and after 6-month follow-up using the commercially available system (Vivid 7, General Electric-Vingmed, USA). Images were obtained using a $3.5 \mathrm{MHz}$ transducer at an appropriate depth. The LVEF was calculated using the biplane Simpson's method from conventional apical four-chamber images. LV end-diastolic diameter (LVEDD) and left atrial diameter (LAD) were measured from M-mode. The area of mitral regurgitation (MR) was assessed semiquantitatively.

2.3. CRT Implantation. CRT was performed with standard techniques via the left subclavian vein. It was a routine procedure to insert the transvenous LV pacing lead into a branch of the coronary sinus. The LV lead was preferentially placed into the lateral or posterolateral vein or an alternative vein if the lateral or posterolateral vein could not be accessed. In summary, the position of the LV lead was determined by the coronary sinus angiographic data. Wherever the LV lead was placed, it was ensured that there were satisfactory pacing parameters without phrenic nerve stimulation. The right atrial lead was placed in the right atrial appendage and the right ventricular lead was placed in the right ventricular apex conventionally.

2.4. Patients' Follow-Up, Determination of Super-Response, and End Points. First follow-up visit was scheduled 1 month after CRT implantation and every 6 months thereafter. Echocardiography and HF symptoms were assessed at 6-month follow-up. Patients were considered as superresponders after 6-month follow-up when NYHA class reduction to I or II combined with near normalization of LVEF (defined as LVEF $\geq 50 \%$ ) was observed. All-cause mortality or hospitalization for heart failure was referred to combined end point.
2.5. Statistical Analysis. Continuous variables were expressed as mean \pm standard deviation or medians (25th-75th percentile) according to their normality following Kolmogorov-Smirnov test. Categorical variables were presented as count and percentage. Differences between groups were analyzed using Student's t-test for parametric variable, MannWhitney $U$ test for nonparametric variables, and chi-square test for categorical variables. Intergroup comparisons were performed with paired t-test. Only variables with $\mathrm{P}<0.1$ in the univariate logistic regression were entered into multivariate logistic regression analysis to identify independent predictors of super-response using backward stepwise procedure. Receiver operator characteristic (ROC) curve was used for evaluation of continuous variables to predict superresponse, and the Youden index was used to determine the optimal cut-off value. Kaplan-Meier curves, with Log Rank P test, compared all-cause death or HF hospitalization. Multivariate Cox proportional hazards models were constructed to identify independent predictors of combined end point, using the variables with $\mathrm{P}<0.1$ in the univariate Cox proportional hazards models. A two-sided $\mathrm{P}$ value $<0.05$ was considered statistically significant. SPSS 19.0 (SPSS Inc., USA) and MedCalc 18.6.0 (MedCalc Inc., Belgium) were used to perform the statistical analysis.

\section{Results}

3.1. Baseline Characteristics of the Study Patients. In the present study, a total of 73 patients ( 21 females; mean age $60.32 \pm 9.78$ years) who fulfilled the inclusion criteria were enrolled. 46 patients had LBBB, 2 patients had right bundle branch block (RBBB), and 16 patients had intraventricular conduction delay (IVCD). Moreover, 1 patient upgraded from dual-chamber pacemaker (DDD) and 2 patients replaced CRT. Of all patients, 17 patients $(23.3 \%)$ were considered as super-responders (NYHA class I or II, LVEF $\geq 50 \%$ ); others were therefore regarded as non-super-responders. There were no significant differences with regard to age, gender, NYHA class, hypertension, diabetes, atrial fibrillation, ischemic etiology, LVEF, LVEDD, MR, QRS duration, ACE-I/ARB, and beta-blockers as well as spironolactone between the two groups. However, the super-responders group had shorter HF duration $(\mathrm{P}<0.001)$ and smaller LAD $(\mathrm{P}=0.001)$ and contained more frequent LBBB $(\mathrm{P}=0.020)$ compared with the non-super-responders group (Table 1).

3.2. Predictors of Super-Response to CRT-P/D. As shown in Table 2, HF duration, LAD, and LBBB at baseline were associated with super-response to CRT-P/D in univariate analysis. Multivariate analysis including these variables and MR (univariate analysis, $\mathrm{P}<0.1$ ) revealed that $\mathrm{HF}$ duration (OR: 0.95, 95\% CI: 0.92-0.98, P = 0.002), LAD (OR: 0.72, 95\% CI: $0.58-0.89, \mathrm{P}=0.002$ ), and LBBB (OR: $10.91,95 \% \mathrm{CI}$ : $1.30-91.64, \mathrm{P}=0.028$ ) were independent predictors of superresponse to CRT-P/D.

Receiver operating characteristic (ROC) curve analysis was performed to evaluate the value of HF duration, LAD and LBBB in predicting super-response to CRT. The area under the curve (AUC) for baseline HF duration was 0.789 
TABLE 1: Baseline characteristics of patients between super-responders and non-super-responders.

\begin{tabular}{|c|c|c|c|c|}
\hline characteristics & $\begin{array}{l}\text { Total population } \\
\qquad(\mathrm{n}=73)\end{array}$ & $\begin{array}{l}\text { super-responders } \\
\qquad(\mathrm{n}=17)\end{array}$ & $\begin{array}{l}\text { non-super-responders } \\
\qquad(\mathrm{n}=56)\end{array}$ & $P$ Value \\
\hline Age (years) & $60.32 \pm 9.78$ & $60.00 \pm 9.87$ & $61.35 \pm 9.72$ & 0.621 \\
\hline Gender (female) & $21(28.8 \%)$ & $7(41.2 \%)$ & $14(25.0 \%)$ & 0.197 \\
\hline NYHA class & $3(3-3)$ & $3(2-3)$ & $3(3-3)$ & 0.285 \\
\hline HF duration (months) & $48(18-78)$ & $12(6-60)$ & $60(27-96)$ & $<0.001$ \\
\hline Hypertension, n (\%) & $16(21.9 \%)$ & $5(29.4 \%)$ & $11(19.6 \%)$ & 0.394 \\
\hline Diabetes, n (\%) & $8(11.0 \%)$ & $3(17.6 \%)$ & $5(8.9 \%)$ & 0.378 \\
\hline Atrial fibrillation, n (\%) & $9(12.3 \%)$ & $1(5.9 \%)$ & $8(14.3 \%)$ & 0.675 \\
\hline Ischemic etiology, n (\%) & $12(16.4 \%)$ & $4(23.5 \%)$ & $8(14.3 \%)$ & 0.456 \\
\hline LVEF (\%) & $32(27-34)$ & $32.6(28-35)$ & $31(25-33.75)$ & 0.140 \\
\hline $\mathrm{LAD}(\mathrm{mm})$ & $45(39.75-47)$ & $40(38-42)$ & $46(41.23-48)$ & 0.001 \\
\hline LVEDD (mm) & $70.22 \pm 7.98$ & $68.25 \pm 8.37$ & $70.82 \pm 7.84$ & 0.247 \\
\hline $\operatorname{MR}\left(\mathrm{cm}^{2}\right)$ & $7.0(4.5-9.98)$ & $5.99(4.30-7.55)$ & $7.32(4.5-10.41)$ & 0.079 \\
\hline QRS duration (ms) & $164.0 \pm 20.99$ & $171.2 \pm 16.54$ & $161.9 \pm 21.83$ & 0.109 \\
\hline LBBB, n (\%) & $46(63.0 \%)$ & $15(88.2 \%)$ & $31(55.4 \%)$ & 0.020 \\
\hline ACE-I/ARB, n (\%) & $67(91.8 \%)$ & $16(94.1 \%)$ & $51(91.1 \%)$ & 1.000 \\
\hline Beta-blockers, n (\%) & $66(90.4 \%)$ & $16(94.1 \%)$ & $50(89.3 \%)$ & 1.000 \\
\hline Spironolactone, n (\%) & $61(83.6 \%)$ & $15(88.2 \%)$ & $46(82.1 \%)$ & 0.720 \\
\hline
\end{tabular}

Values are mean $\pm \mathrm{SD}$, median (range), or $\mathrm{n}(\%)$.

NYHA: New York Heart Association; HF: Heart Failure; LVEF: left ventricular ejection fraction; LAD: left atrial dimension; LVEDD: left ventricular enddiastolic dimension; MR: mitral regurgitation; LBBB: left bundle branch block; ACEI: angiotensin-converting-enzyme inhibitor; ARB: angiotensin receptor blocker.

TABLE 2: Univariate and multivariate logistic regression analyses of predictors of super-response.

\begin{tabular}{|c|c|c|c|c|}
\hline \multirow[b]{2}{*}{ Baseline characteristics } & \multicolumn{2}{|c|}{ Univariate analysis } & \multicolumn{2}{|c|}{ Multivariate analysis } \\
\hline & OR (95\% CI) & $P$ Value & OR (95\% CI) & $P$ Value \\
\hline Age & $1.02(0.96-1.07)$ & 0.616 & & \\
\hline Gender & $2.10(0.67-6.56)$ & 0.202 & & \\
\hline NYHA class & $0.61(0.25-1.49)$ & 0.274 & & \\
\hline HF duration & $0.97(0.94-0.99)$ & 0.003 & $0.95(0.92-0.98)$ & 0.002 \\
\hline Hypertension & $1.71(0.50-5.86)$ & 0.397 & & \\
\hline Diabetes & $2.19(0.47-10.29)$ & 0.322 & & \\
\hline Atrial fibrillation & $0.38(0.04-3.23)$ & 0.372 & & \\
\hline Ischemic etiology & $1.85(0.48-7.10)$ & 0.373 & & \\
\hline LVEF & $1.10(0.96-1.26)$ & 0.186 & & \\
\hline LAD & $0.83(0.74-0.94)$ & 0.003 & $0.72(0.58-0.89)$ & 0.002 \\
\hline LVEDD & $0.96(0.89-1.03)$ & 0.246 & & \\
\hline MR & $0.88(0.75-1.02)$ & 0.081 & $1.21(0.89-1.65)$ & 0.223 \\
\hline QRS duration & $1.02(1.00-1.05)$ & 0.115 & & \\
\hline LBBB & $6.05(1.26-28.98)$ & 0.024 & $10.91(1.30-91.64)$ & 0.028 \\
\hline ACE-I/ARB & $1.57(0.17-14.43)$ & 0.691 & & \\
\hline Beta-blockers & $1.92(0.22-17.17)$ & 0.559 & & \\
\hline Spironolactone & $1.63(0.32-8.29)$ & 0.556 & & \\
\hline
\end{tabular}

NYHA: New York Heart Association; HF: heart failure; LVEF: left ventricular ejection fraction;

LAD: left atrial dimension; LVEDD: left ventricular end-diastolic dimension; MR: mitral regurgitation; LBBB: left bundle branch block; ACE-I: angiotensinconverting-enzyme inhibitor; ARB: angiotensin receptor blocker

OR: odds ratio; 95\% CI: 95\% confidence interval.

(95\% CI: 0.67-0.91, $\mathrm{P}<0.001$ ), with HF duration $\leq 36$ months having $88 \%$ sensitivity and $70 \%$ specificity. Baseline LAD $\leq$ $42 \mathrm{~mm}$ was $82 \%$ sensitive and $71 \%$ specific for predicting super-response to CRT (AUC 0.775; 95\% CI: 0.67-0.88; P
$<0.001)$. For combination of HF duration and LAD, the AUC was 0.899 (95\% CI: 0.83-0.98, $\mathrm{P}<0.001$ ), with $\mathrm{HF}$ duration $\leq 48$ months together with $\mathrm{LAD} \leq 42 \mathrm{~mm}$ having $88 \%$ sensitivity and $79 \%$ specificity. There was no significant 
TABLE 3: Area under the curve and sensitivity and specificity for HF duration, LAD, and combination of HF duration and LAD.

\begin{tabular}{|c|c|c|c|c|c|c|}
\hline Variables & AUC & $95 \%$ CI & $P$ for AUC & Cut-off value & $\begin{array}{c}\text { Sensitivity } \\
(\%)\end{array}$ & $\begin{array}{c}\text { Specificity } \\
(\%)\end{array}$ \\
\hline HF duration (months) & 0.789 & $0.67-0.91$ & $<0.001$ & $\leq 36$ & 88 & 70 \\
\hline $\mathrm{LAD}(\mathrm{mm})$ & 0.775 & $0.67-0.88$ & $<0.001$ & $\leq 42$ & 82 & 71 \\
\hline $\begin{array}{l}\text { Combination of HF } \\
\text { duration and LAD } \\
\text { (months, } \mathrm{mm} \text { ) }\end{array}$ & 0.899 & $0.83-0.98$ & $<0.001$ & $\leq 48$ and $\leq 42$ & 88 & 79 \\
\hline
\end{tabular}

HF: heart failure; LAD: left atrial dimension; AUC: the area under the curve; $95 \%$ CI: $95 \%$ confidence interval.

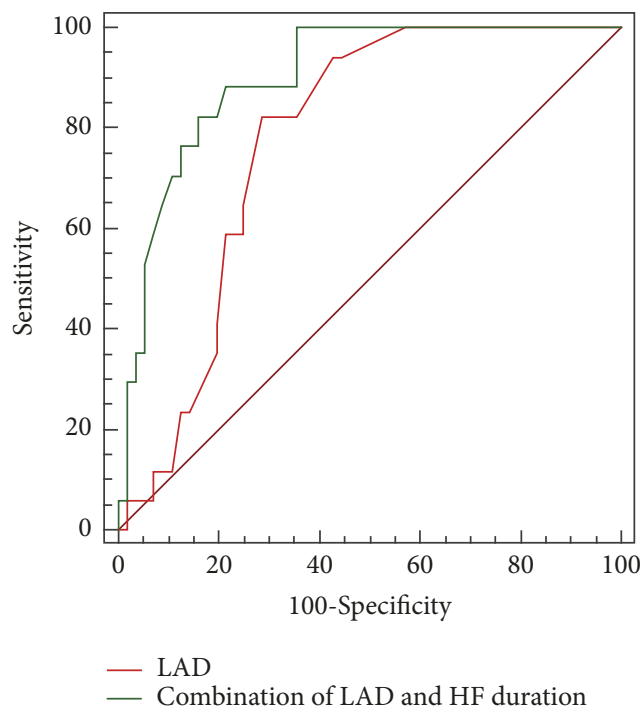

(a) $\operatorname{AUC}(0.899$ versus $0.775, \mathrm{Z}=2.487, \mathrm{P}=0.013)$

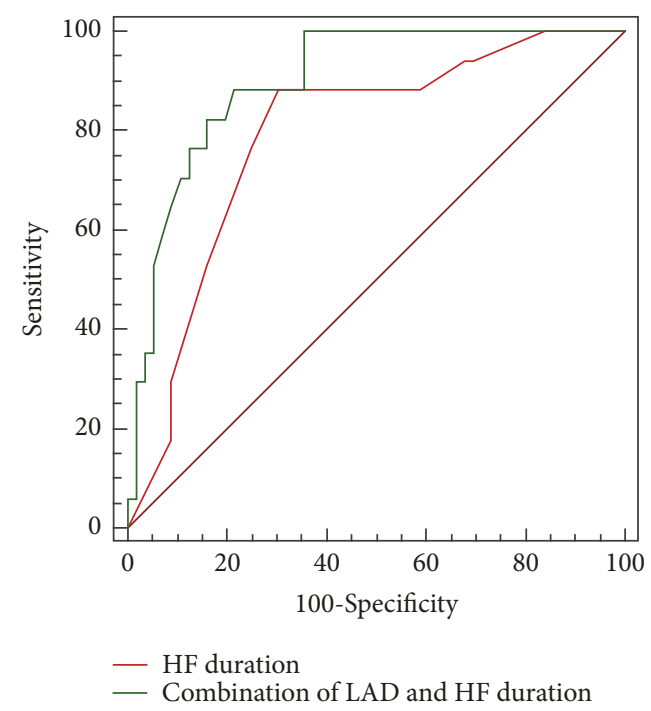

(b) AUC (0.899 versus 0.789, $\mathrm{Z}=2.207, \mathrm{P}=0.027)$

FIGURE 1: (a) ROC curve for LAD (red line) and combination of HF duration and LAD (green line) in predicting super-response; (b) ROC curve for HF duration (red line) and combination of HF duration and LAD (green line) in predicting super-response. ROC: receiver operator characteristic; HF: heart failure; LAD: left atrial dimension; AUC: the area under the curve.

difference between the AUC of HF duration and LAD $(0.789$ versus $0.775, \mathrm{Z}=0.190, \mathrm{P}=0.849)$. Combination of $\mathrm{HF}$ duration and LAD significantly increased the AUC compared with standalone HF duration (0.899 versus $0.789, \mathrm{Z}=2.207$, $\mathrm{P}=0.027)$ or standalone LAD (0.899 versus $0.775, \mathrm{Z}=2.487$, $\mathrm{P}=0.013$ ) (Figure 1, Table 3).

3.3. Six-Month Follow-Up after CRT. Three patients died within 6 months after CRT in the non-super-responders group. Therefore, a total of 70 patients completed the echocardiographic, clinic visit, or telephone surveys at 6-month follow-up. Patients' characteristics including cardiac function and echocardiographic data at baseline and follow-up in two groups are summarized in Table 4. After 6-month followup, there were significant improvements of NYHA class, LVEF, LVEDD, and MR in both groups $(\mathrm{P}<0.05$ for all comparisons). Compared with non-super-responders group, the super-responders group demonstrated significantly better outcome as indicated by better improvement in cardiac function $(\mathrm{P}<0.001)$, higher $\operatorname{LVEF}(\mathrm{P}<0.001)$, smaller LVEDD $(\mathrm{P}<0.001)$, and MR $(\mathrm{P}<0.001)$ at 6 -month followup.
3.4. Long-Term Prognosis. During a mean follow-up of 22.56 \pm 10.38 months, the combined end point of hospitalization for $\mathrm{HF}$ or all-cause death occurred in 17 patients. In the super-responders group, 1 (5.9\%) hospitalization for $\mathrm{HF}$ and $0(0 \%)$ deaths were reported, compared with $10(17.9 \%)$ hospitalizations for $\mathrm{HF}$ and $6(10.7 \%)$ deaths in the nonsuper-responders group. As shown in the Kaplan-Meier curves (Figure 2), the cumulative incidences of combined end point were significantly lower in the super-responders group, $\mathrm{LAD} \leq 42 \mathrm{~mm}$ group, and combination of HF duration $\leq 48$ months and LAD $\leq 42 \mathrm{~mm}$ group compared with the nonsuper-responders group (Log Rank $\mathrm{P}=0.044)$, LAD $>42 \mathrm{~mm}$ group ( $\log \operatorname{Rank} \mathrm{P}=0.015)$, and others $(\log \operatorname{Rank} \mathrm{P}=0.021)$, respectively. However, no statistically significant difference was observed between the HF duration $\leq 36$ months group and the HF duration $>36$ months group for the combined end point ( $\log \operatorname{Rank} \mathrm{P}=0.054)$.

3.5. Predictors of the Combined End Point. A multivariate Cox regression model was carried out adjusting for LAD, LVEDD, and LBBB (univariate regression model analysis, $\mathrm{P}<0.1)$. LBBB remained associated with a lowered risk of 
TABLE 4: Comparison of cardiac function and echocardiographic data at baseline and follow-up in two groups.

\begin{tabular}{|c|c|c|c|c|c|c|c|}
\hline \multirow{2}{*}{ Variables } & \multicolumn{3}{|c|}{ super-responders $(\mathrm{n}=17)$} & \multicolumn{3}{|c|}{ non-super-responders $(n=56)$} & \multirow{2}{*}{$P *$ Value } \\
\hline & baseline & follow-up & $P$ Value & baseline & follow-up & $P$ Value & \\
\hline NYHA class & $3(2-3)$ & $2(1-2)$ & $<0.001$ & $3(3-3)$ & $2(2-3)$ & $<0.001$ & $<0.001$ \\
\hline LVEF (\%) & $32.6(28-35)$ & $57(54-59)$ & $<0.001$ & $31(25-33.8)$ & $34.5(29.3-38.8)$ & 0.003 & $<0.001$ \\
\hline $\operatorname{LVEDD}(\mathrm{mm})$ & $68.3 \pm 8.4$ & $55.3 \pm 6.1$ & $<0.001$ & $70.8 \pm 7.8$ & $68.7 \pm 9.5$ & 0.034 & $<0.001$ \\
\hline $\operatorname{MR}\left(\mathrm{cm}^{2}\right)$ & $6.0(4.3-7.6)$ & $1(0-2.4)$ & $<0.001$ & $7.3(4.5-10.4)$ & $4.6(2.5-7.3)$ & $<0.001$ & $<0.001$ \\
\hline
\end{tabular}

NYHA: New York Heart Association; LVEF: left ventricular ejection fraction; LVEDD: left ventricular end-diastolic dimension; MR: mitral regurgitation. $P *$ : follow-up between two groups.

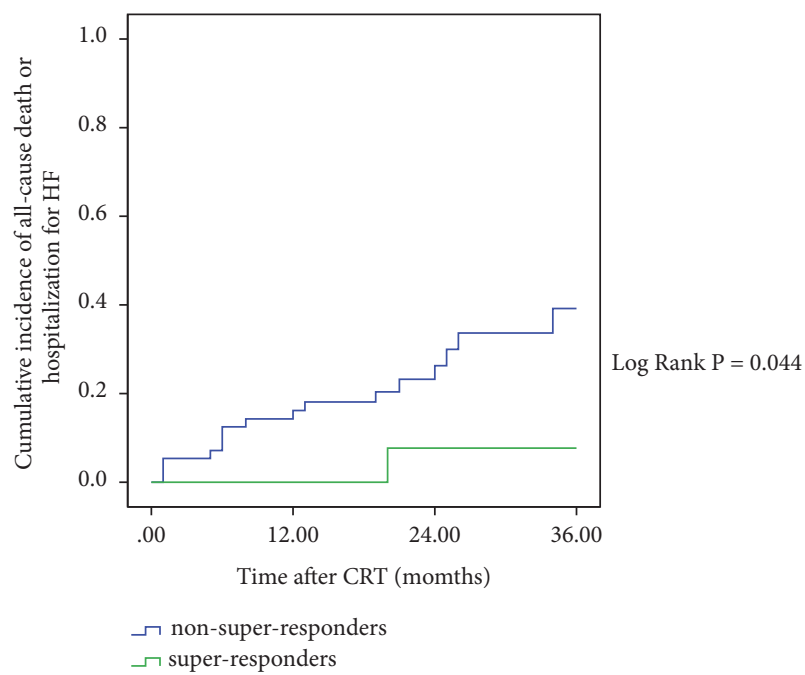

(a)

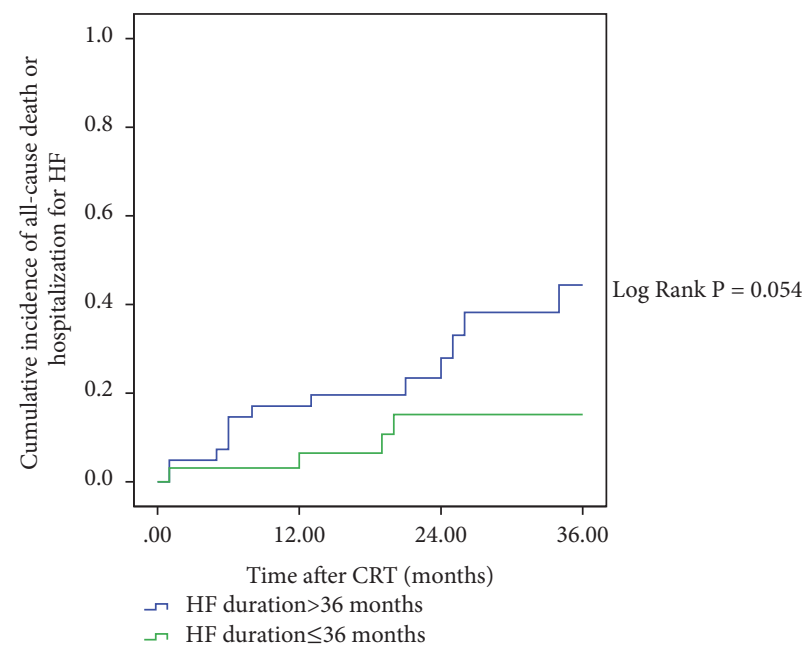

(c)

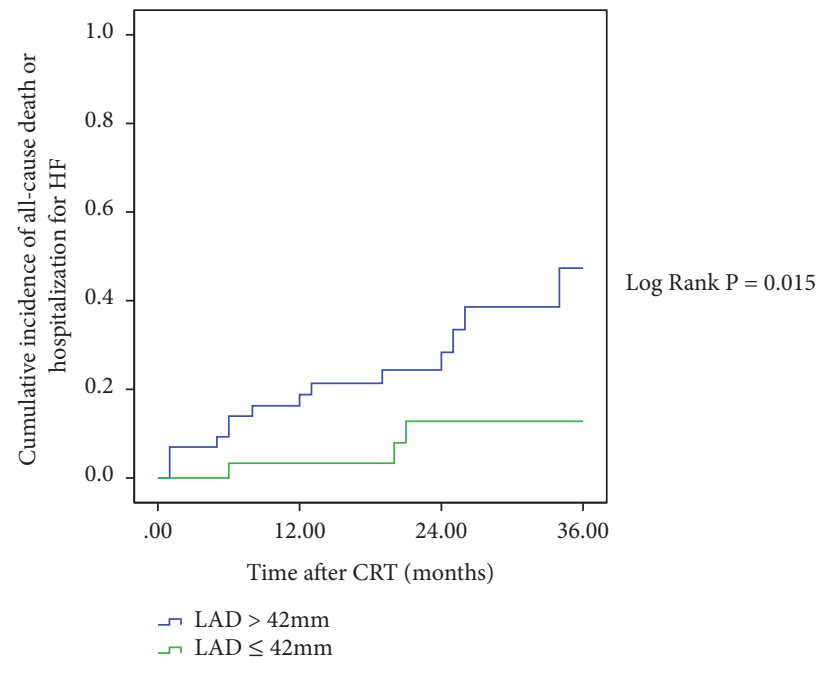

(b)

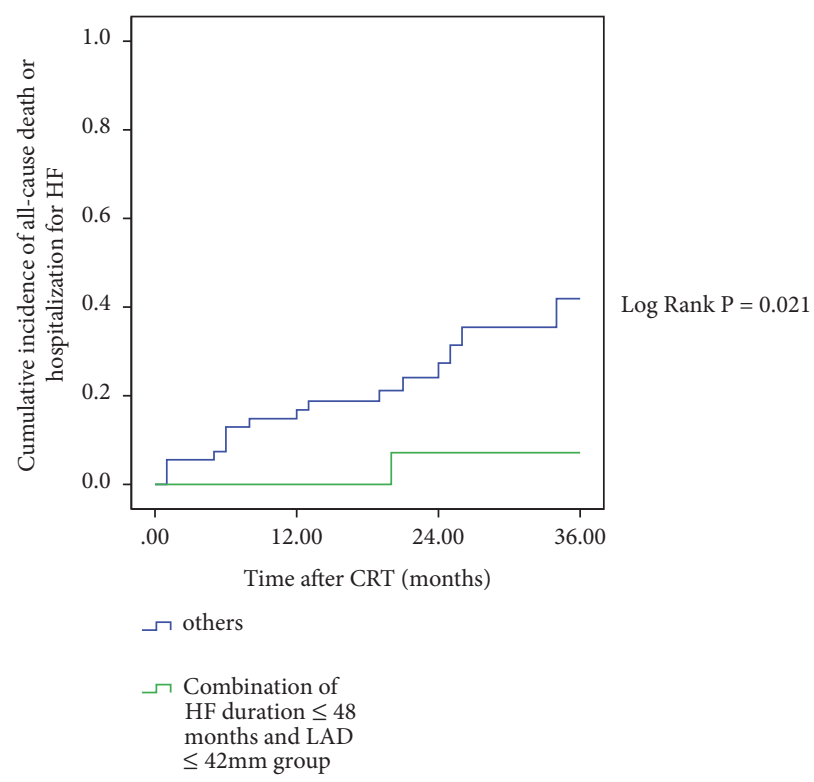

(d)

Figure 2: Four Kaplan-Meier curves (response category, LAD, HF duration, and combination of HF duration and LAD) of cumulative incidence of all-cause death or hospitalization for heart failure. (a) For the combined end point, super-responders (green line) performed the best compared to non-super-responders (blue line). (b) For the combined end point, LAD $\leq 42 \mathrm{~mm}$ group (green line) performed the best compared to LAD $>42 \mathrm{~mm}$ group (blue line). (c) For the combined end point, there was no difference between HF duration $\leq 36 \mathrm{months}$ group and HF duration $>36$ months group. (d) For the combined end point, combination of HF duration $\leq 48$ months and LAD $\leq 42 \mathrm{~mm}$ group (green line) performed the best compared to others (blue line). HF: heart failure; LAD: left atrial dimension. 
Table 5: Multivariate Cox regression models for combined end point.

\begin{tabular}{llll}
\hline Variables & HR & $95 \%$ CI & $P$ Value \\
\hline LAD $(\mathrm{mm})$ & 1.09 & $1.02-1.17$ & 0.014 \\
LBBB & 0.19 & $0.07-0.57$ & 0.003 \\
\hline
\end{tabular}

LAD: left atrial dimension; LBBB: left bundle branch block.

95\% CI: 95\% confidence interval; HR: hazard ratio.

the combined end point (HR: $0.19,95 \% \mathrm{CI}: 0.07-0.57, \mathrm{P}=$ 0.003 ), whereas LAD was associated with a raised risk of the combined end point (HR: 1.09, 95\% CI: 1.02-1.17, P = 0.014) (Table 5).

\section{Discussion}

The main findings of our study were as follows: (1) HF duration, LAD, and LBBB were independent predictors of superresponse to CRT; (2) the combination of HF duration and LAD could provide better prediction of CRT super-response; (3) super-responders had excellent LV reverse remodeling and clinical prognosis during follow-up; (4) the cumulative incidences of combined end point were significantly lower in the super-responders group, LAD $\leq 42 \mathrm{~mm}$ group, and combination of HF duration $\leq 48$ months and LAD $\leq 42 \mathrm{~mm}$ group compared with the non-super-responders group, LAD $>42 \mathrm{~mm}$ group, and others, respectively; (5) LBBB showed a decreased risk for combined end point, whereas LAD was associated with an increased risk of combined end point.

LVEF was used to diagnose $\mathrm{HF}$ and evaluate the prognosis of HF treatments. Reduced LVEF was associated with poor survival [9]. Moreover, most clinical trials and guidelines for CRT-P/D implantation are based on measurement of LVEF to select patients. Hence, LVEF was regarded as one of the most widely used measurement to define CRT super-response. In our study, the definition of super-response included the combination of NYHA class (I-II) and LVEF $\geq 50 \%$. Previous studies reported an incidence of $12 \%$ to $37.8 \%$ of CRT superresponse [9]. We found an incidence of $23.3 \%$ of CRT superresponse based on our definition of super-response, similar to the previous studies by Castellant et al. (21.5\%)[10] and Gasparini et al. (26\%)[11] using the same definition.

In the MADIT-CRT study by Hsu et al. of 752 CRT recipients, smaller LA volume was independently associated with super-response to CRT therapy [5]. Similarly, Reant et al. demonstrated that LA volume $<55 \mathrm{ml}$ could independently predict CRT super-response. In our study, LAD could be independently predictive of super-response, aligned with the above two studies. Moreover, using ROC curves, LAD $\leq 42$ $\mathrm{mm}$ had $82 \%$ sensitivity and $71 \%$ specificity for predicting super-response to CRT (AUC 0.775; 95\% CI: 0.67-0.88; P $<0.001)$. We also found $\mathrm{LAD} \leq 42 \mathrm{~mm}$ was associated with improved clinical outcome and increased LAD tended to be an increased risk factor of the combined end point (HR: 1.09, 95\% CI: 1.02-1.17, P = 0.014). LAD was a sensitive marker of chronic HF. Previous studies had reported that increased LA size was associated with cardiovascular morbidity and mortality in HF patients $[12,13]$. In addition, LAD was correlated with changes in electrophysiological characteristics, such as interatrial conduction times and functional LA conduction blocks $[8,14]$. Hence, enlarged LA limited positive effects of CRT and contributed progression of HF. In other words, better clinical outcome and response were associated with smaller LA. Further investigations were justified to clarify the pathophysiological mechanisms of enlarged LA influencing outcomes in patients receiving CRT and whether addressing these mechanisms could improve the response to CRT.

In the present study, HF duration was an independent predictor of super-response to CRT, with $\mathrm{HF}$ duration $\leq 36$ months having $88 \%$ sensitivity and $70 \%$ specificity (AUC, 0.789, 95\% CI: 0.67-0.91, $\mathrm{P}<0.001$ ), in line with a previous study which showed that HF duration $\leq 12$ months remained significantly associated with super-response in multivariate analysis [15]. However, our present results showed that although there was no statistical difference, HF duration $\leq 36$ months tended to decrease the occurrence of combined end point. To our knowledge, it was the first study to evaluate the value of combination of HF duration and LAD in predicting super-response. The result showed that the combination of HF duration and LAD could provide better performance than HF duration alone ( 0.899 versus $0.789, \mathrm{Z}=2.207, \mathrm{P}=0.027$ ) or LAD alone (0.899 versus $0.775, \mathrm{Z}=2.487, \mathrm{P}=0.013)$ in prediction of super-response. It may indicate that CRT superresponse could occur more easily in the earlier phases of HF. More researches were needed to confirm our results in the future.

Abnormal left ventricular activation sequence in LBBB might lead to electrical and mechanical dyssynchrony [16]. Thus, CRT targeting resynchronization of the delayed left ventricle was recommended for HF patients with LBBB [17]. The previous study, which enrolled 233 patients, 101 patients (43.3\%) with LBBB, 68.8\% (22) in super-responders, and $39.3 \%$ (79) in non-super-responders, reported that only LBBB was significantly associated with super-response in multivariate analysis [18]. Hsu et al. also found that LBBB could independently predict super-response to CRT therapy, which enrolled 752 patients, 534 patients (71.0\%) with LBBB, $55.8 \%$ (106) in hyporesponders, $70.9 \%$ (263) in responders, and $86.4 \%$ (165) in super-responders[5]. In our study, 46 patients $(63.0 \%)$ had LBBB, $88.2 \%$ (15) in super-responders and $55.4 \%$ (31) in non-super-responders. Similarly, we found that LBBB were independent predictors of super-response to CRT. In addition, a previous study by Tian et al. of 58 CRT recipients showed that true $\mathrm{LBBB}$ was an independent predictor of super-response [19]. Moreover, our study showed that LBBB was associated with a lowered risk of the combined end point (HR: 0.19, 95\% CI: 0.07-0.57, $\mathrm{P}=0.003$ ), in line with the previous MADIT-CRT study by Hsu et al. which demonstrated that baseline LBBB was significantly associated 
with a decreased risk of HF or all-cause death (HR: 0.57; 95\% CI: 0.34 to $0.94 ; \mathrm{p}=0.029$ ) [5].

Several other variables, including female sex, smaller LV, NICM, BMI, and wider QRS duration, were reported to be associated with super-response to CRT. Unfortunately, those variables could not predict super-response in our study, which could be due to differences with the super-response definition adopted and patients enrolled.

In the study, super-responders had greater reductions in LVEDD $(\mathrm{P}<0.001)$ and MR $(\mathrm{P}<0.001)$ than non-superresponders. In other words, super-responders had excellent LV reverse remodeling, which was associated with favourable clinical outcome, in line with a previous study[20]. The incidences of all-cause death and HF hospitalization were $8.2 \%$ and $15.1 \%$, respectively, in our study. In the MADITCRT study, all-cause death occurred in 3.3\% of all patients and the incidence of nonfatal HF event was $6.4 \%$ with a median follow-up of 15.2 months [5]. In addition, one recent study with 347 patients showed all-cause death and HF hospitalization occurred in $23 \%$ and $22 \%$ in all patients who were followed up for a median of 5.3 years, respectively [4]. We found super-response was associated with a reduced occurrence of all-cause death or HF hospitalization, which was consistent with the previous studies $[4,5,18,20$, 21]. Moreover, a previous study reported that the patients considered as super-responders had a significantly reduced incidence of ventricular arrhythmias [22]. Similarly, Killu et al. demonstrated that the super-response to CRT-D group had very low rates of ventricular arrhythmias requiring implantable cardioverter defibrillator (ICD) therapy compared with the non-super-response to CRT-D group [23].

\section{Limitations}

This study has a few limitations. First, the retrospective nature of this study probably resulted in unidentified confounders. Second, patients in our study came from a single center and the sample size was relatively small; therefore, it may not be sufficiently representative. Third, the duration of follow-up in our study was relatively short and therefore predictors of delayed improvement of LVEF could not be evaluated. Last, patients who were lost to follow-up were not included in our study, so there may be patient selection bias. However, those aspects were inherent limitations of the "real world" studies, despite our best efforts to avoid these limitations. Therefore, further large multiple-center prospective trials were needed to confirm our results in the future.

\section{Conclusions}

HF duration, LAD, and LBBB were independent predictors of super-response to CRT. The combination of HF duration and LAD strongly predicted CRT super-response. Superresponders had excellent LV reverse remodeling and clinical prognosis during follow-up. LAD $\leq 42 \mathrm{~mm}$ and the combination of $\mathrm{HF}$ duration $\leq 48$ months and $\mathrm{LAD} \leq 42 \mathrm{~mm}$ decreased the incidences of combined end point. LBBB and LAD were independently associated with all-cause death and HF hospitalization.

\section{Data Availability}

The data used to support the findings of this study are available from the corresponding author upon request.

\section{Conflicts of Interest}

The authors declare that they are no conflicts of interest.

\section{Acknowledgments}

This work was supported by the Clinical Research Fund of Xinqiao Hospital of Third Military Medical University (no. 2014YlC12) and the Starting Fund for Talents Returning from Overseas of Ministry of Education (no. 2015A497).

\section{References}

[1] J. Holzmeister and C. Leclercq, "Implantable cardioverter defibrillators and cardiac resynchronisation therapy," The Lancet, vol. 378, no. 9792, pp. 722-730, 2011.

[2] J. M. Hsing, K. A. Selzman, C. Leclercq et al., "Paced left ventricular QRS width and ECG parameters predict outcomes after cardiac resynchronization therapy," Circulation: Arrhythmia and Electrophysiology, vol. 4, no. 6, pp. 851-857, 2011.

[3] A. M. Killu, A. Mazo, A. Grupper et al., "Super-response to cardiac resynchronization therapy reduces appropriate implantable cardioverter defibrillator therapy," EP Europace, vol. 20, no. 8, pp. 1303-1311, 2018.

[4] A. Ghani, P. P. Delnoy, A. Adiyaman et al., "Predictors and longterm outcome of super-responders to cardiac resynchronization therapy," Clinical Cardiology, vol. 40, no. 5, pp. 292-299, 2017.

[5] J. C. Hsu, S. D. Solomon, M. Bourgoun et al., "Predictors of super-response to cardiac resynchronization therapy and associated improvement in clinical outcome," Journal of the American College of Cardiology, vol. 59, no. 25, pp. 2366-2373, 2012.

[6] R. J. van Bommel, J. J. Bax, W. T. Abraham et al., "Characteristics of heart failure patients associated with good and poor response to cardiac resynchronization therapy: a PROSPECT (Predictors of Response to CRT) sub-analysis," European Heart Journal, vol. 30, no. 20, pp. 2470-2477, 2009.

[7] K. A. Ellenbogen and J. F. Huizar, "Foreseeing super-response to cardiac resynchronization therapy," Journal of the American College of Cardiology, vol. 59, no. 25, pp. 2374-2377, 2012.

[8] L. Stefan, K. Sedlacek, D. Cerna et al., "Small left atrium and mild mitral regurgitation predict super-response to cardiac resynchronization therapy," Europace, vol. 14, no. 11, pp. 16081614, 2012.

[9] A. E. Epstein, J. P. DiMarco, K. A. Ellenbogen et al., "ACC/ AHA/HRS 2008 guidelines for device-based therapy of cardiac rhythm abnormalities: executive summary," Heart Rhythm, vol. 5, no. 6, pp. 934-955, 2008.

[10] P. Castellant, M. Fatemi, E. Orhan, Y. Etienne, and J. J. Blanc, "Patients with non-ischaemic dilated cardiomyopathy and hyper-responders to cardiac resynchronization therapy: characteristics and long-term evolution," Europace, vol. 11, no. 3, pp. 350-355, 2009.

[11] M. Gasparini, F. Regoli, C. Ceriotti et al., "Remission of left ventricular systolic dysfunction and of heart failure symptoms after cardiac resynchronization therapy: Temporal pattern and clinical predictors," American Heart Journal, vol. 155, no. 3, pp. 507-514, 2008. 
[12] A. Rossi, M. Cicoira, L. Zanolla et al., "Determinants and prognostic value of left atrial volume in patients with dilated cardiomyopathy," Journal of the American College of Cardiology, vol. 40, no. 8, pp. 1425-1430, 2002.

[13] P. Giannuzzi, P. L. Temporelli, E. Bosimini et al., "Independent and incremental prognostic value of doppler-derived mitral deceleration time of early filling in both symptomatic and asymptomatic patients with left ventricular dysfunction," Journal of the American College of Cardiology, vol. 28, no. 2, pp. 383390, 1996.

[14] F. Raybaud, J. P. Camous, P. Benoit, C. Dolisi, and M. Baudouy, "Relationship between interatrial conduction times and left atrial dimension in patients undergoing atrioventricular stimulation," Pacing and Clinical Electrophysiology, vol. 18, no. 3, Pt 1, pp. 447-450, 1995.

[15] N. Antonio, R. Teixeira, L. Coelho et al., "Identification of 'super-responders' to cardiac resynchronization therapy: the importance of symptom duration and left ventricular geometry," Europace, vol. 11, no. 3, pp. 343-349, 2009.

[16] A. Auricchio, C. Fantoni, F. Regoli et al., "Characterization of left ventricular activation in patients with heart failure and left bundle-branch block," Circulation, vol. 109, no. 9, pp. 1133-1139, 2004.

[17] P. Ponikowski, A. A. Voors, S. D. Anker, H. Bueno, J. G. Cleland, and A. J. Coats, "2016 ESC guidelines for the diagnosis and treatment of acute and chronic heart failure: the task Force for the diagnosis and treatment of acute and chronic heart failure of the European Society of Cardiology (ESC). Developed with the special contribution of the Heart Failure Association (HFA) of the ESC," European Heart Journal, vol. 18, no. 8, pp. 891-975, 2016.

[18] J. Rickard, D. J. Kumbhani, Z. Popovic et al., "Characterization of super-response to cardiac resynchronization therapy," Heart Rhythm, vol. 7, no. 7, pp. 885-889, 2010.

[19] Y. Tian, P. Zhang, X. Li et al., "True complete left bundle branch block morphology strongly predicts good response to cardiac resynchronization therapy," EP Europace, vol. 15, no. 10, pp. 1499-1506, 2013.

[20] J. Steffel, G. Milosevic, A. Hurlimann et al., "Characteristics and long-term outcome of echocardiographic super-responders to cardiac resynchronisation therapy: 'real world' experience from a single tertiary care centre," Heart, vol. 97, no. 20, pp.1668-1674, 2011.

[21] E. Adelstein, D. Schwartzman, J. Gorcsan III, and S. Saba, "Predicting hyperresponse among pacemaker-dependent nonischemic cardiomyopathy patients upgraded to cardiac resynchronization," Journal of Cardiovascular Electrophysiology, vol. 22, no. 8, pp. 905-911, 2011.

[22] I. García-Lunar, V. Castro-Urda, J. Toquero-Ramos et al., "Arritmias ventriculares en superrespondedores a la terapia de resincronización cardiaca," Revista Española de Cardiología, vol. 67, no. 11, pp. 883-889, 2014.

[23] A. M. Killu, A. Grupper, P. A. Friedman et al., "Predictors and outcomes of "super-response" to cardiac resynchronization therapy," Journal of Cardiac Failure, vol. 20, no. 6, pp. 379-386, 2014. 


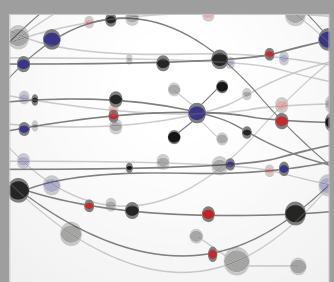

The Scientific World Journal
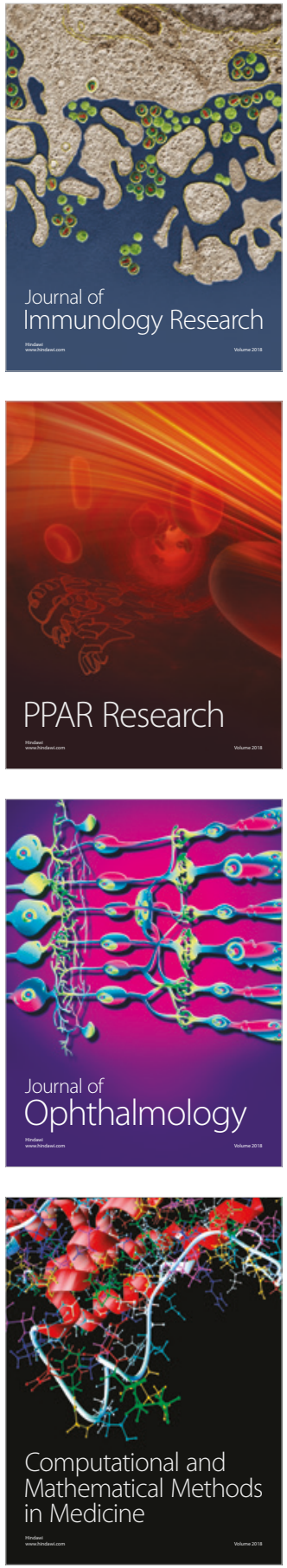

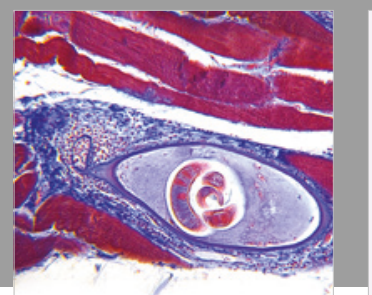

Gastroenterology Research and Practice

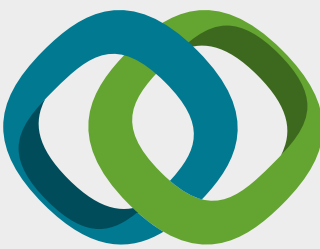

\section{Hindawi}

Submit your manuscripts at

www.hindawi.com
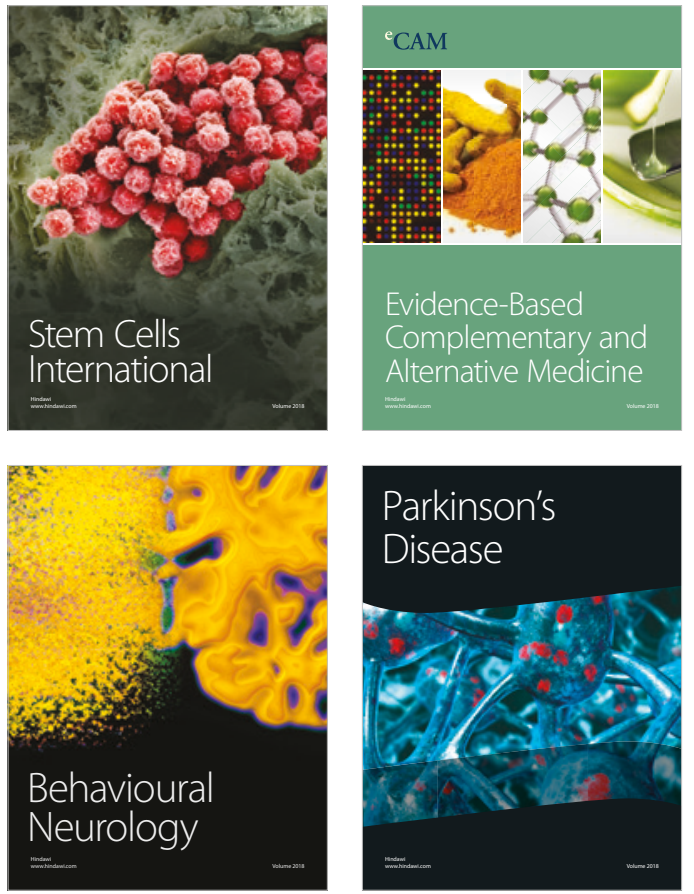

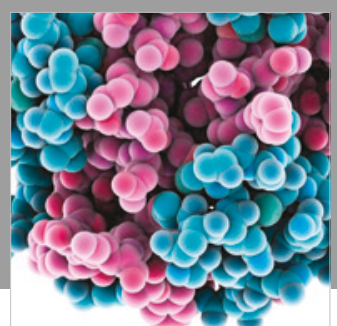

ournal of

Diabetes Research

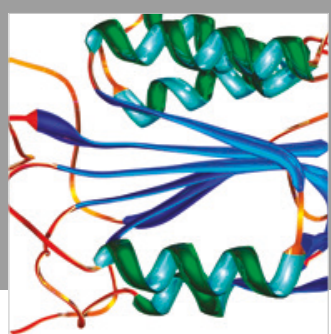

Disease Markers
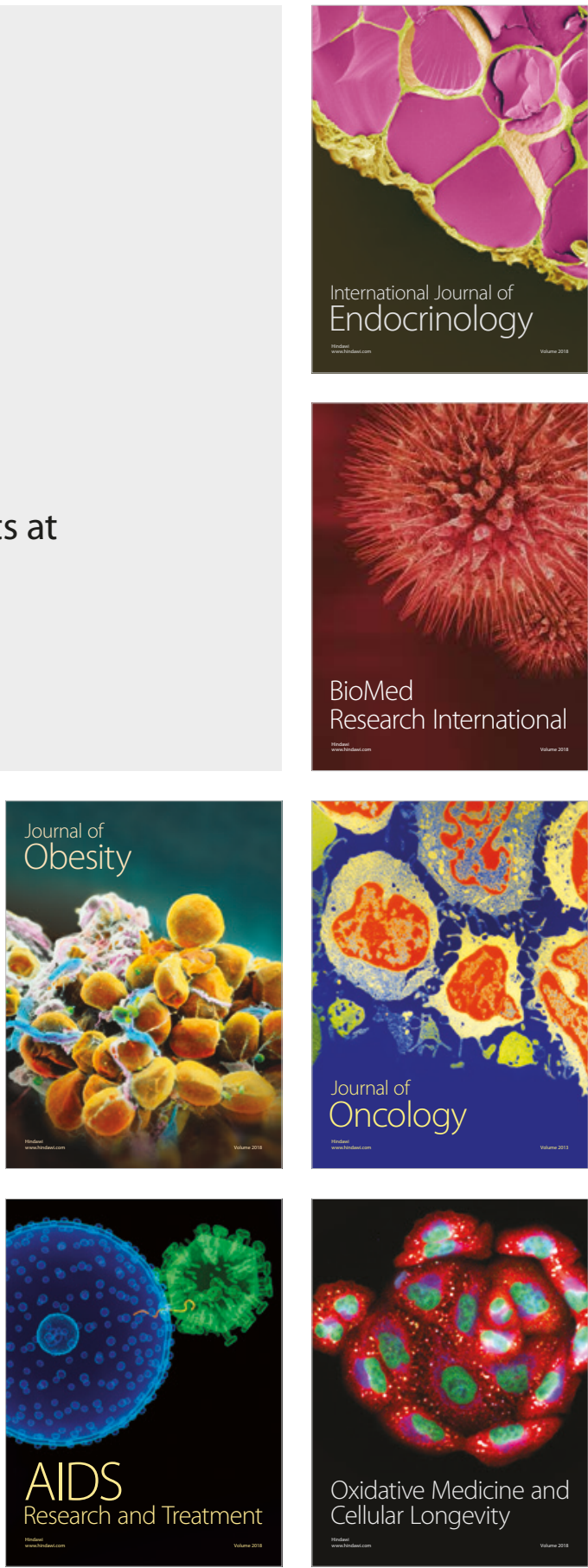